\title{
Disbond detection using guided wave PZT excitation in honeycomb composite sandwich structure (withdrawal notice)
}

\section{Pol, S. Banerjee}

C. B. Pol, S. Banerjee, "Disbond detection using guided wave PZT excitation in honeycomb composite sandwich structure (withdrawal notice)," Proc. SPIE 9063, Nondestructive Characterization for Composite Materials, Aerospace Engineering, Civil Infrastructure, and Homeland Security 2014, 906325 (9 March 2014); doi: 10.1117/12.2042745

Event: SPIE Smart Structures and Materials + Nondestructive Evaluation and Health Monitoring, 2014, San Diego, California, United States 


\section{Disbond detection using guided wave PZT excitation in honeycomb composite sandwich structure (withdrawal notice)}

Proc. SPIE 9063, $906325(2014)$

Online Publication Date: 9 March 2014

Withdrawn from Publication: 25 March 2014

Conference Date: 10-13 March 2014

Conference Location: San Diego, California, United States

Conference Title: Nondestructive Characterization for Composite Materials, Aerospace Engineering, Civil Infrastructure, and Homeland Security 2014

Conference Chairs: H. Felix Wu, Tzu-Yang Yu, Andrew L. Gyekenyesi, Aaron A. Diaz, Peter J. Shull

Pol C.B., and S. Banerjee

Indian Institute of Technology Bombay (India)

This paper has been withdrawn by the publisher because it was not presented at the conference.

Nondestructive Characterization for Composite Materials, Aerospace Engineering, Civil Infrastructure, and Homeland 